International Journal of Biology, Pharmacy and Allied Seiences (IJBPAS)

'A Bridge Betuen Caboratory and QRendo'

WwW.iibpas.com

\title{
PARTIAL PURIFICATION AND QUORUM SENSING INHIBITION OF SYZYGIUM AROMATICUM ON PSEUDOMONAS AERUGINOSA - AN IN VITRO AND IN SILICO APPROACH
}

\author{
ALLURI N, MUTUNGWA W AND MAJUMDAR M* \\ Department of Biotechnology, School of Sciences, Jain University, \#18/3 $9^{\text {th }}$ main, $3^{\text {rd }}$ Block, \\ Jayanagar, Bengaluru, India-560011 \\ *Corresponding Author: Dr. Mala Majumdar: Email: mala.majumdar@jainuniversity.ac.in;
}

Telephone: 080-43226500; Fax: 080-43226507

Received $1^{\text {st }}$ Oct. 2017; Revised $5^{\text {th }}$ Nov. 2017; Accepted 10 ${ }^{\text {th }}$ December 2017; Available online $1^{\text {st }}$ March 2018

\begin{abstract}
Syzygium aromaticum, a popular ethanomedicinal herb, possesses quorum quenching and inhibition of virulence factors of Pseudomonas aeruginosa. The objective of the present study was to partially purify and study quorum sensing inhibition of S.aromaticum methanolic extract using in vitro and in silico docking studies. S. aromaticum was extracted with methanol using Soxhlet apparatus and was subjected to TLC-bioautography using Chromobacterium violaceum as reporter strain. The TLC purified fraction was subjected to biofilm inhibition on P. aeruginosa and GC-MS analysis. The identified compounds from GC-MS were docked with LasI and EsaI proteins. TLC-bioautography analysis showed clear zone of inhibition at the bioactive fraction $\left(\mathrm{R}_{\mathrm{f}}=0.78\right)$ and also exhibited dose dependent (50$200 \mu \mathrm{g} / \mathrm{mL}$ ) biofilm inhibition on P. aeruginosa. GC-MS analysis of active fraction revealed six major compounds viz. 4-hydroxy-3-methoxy-benzaldehyde, 1-phenoxy ethanol, eugenol, chavicol, p-hydroxyacetophenone and 4-azido-phenol that may account for the antibiofilm activity. These compounds were studied for their in silico binding patterns with quorum sensing regulated proteins, LasI and EsaI. Among all, chavicol formed three hydrogen bonds with LasI $(-6.20 \mathrm{kcal} / \mathrm{mol})$ and three hydrogen bonds with EsaI $(-4.36 \mathrm{kcal} / \mathrm{mol})$, indicating best interaction with targeted protein binding sites. The current study demonstrated that the
\end{abstract}


bioactive fraction has the ability of quorum quenching which is supported by in silico analysis and might leads to in vivo establishment in future.

\section{Keywords: Syzygium aromaticum, in silico, TLC-bioautography, LasI, EsaI}

\section{INTRODUCTION}

In the infectious diseases, at least $65 \%$ are linked to the bacterial communities which proliferate by forming biofilms [1]. Bacterial biofilm is the matrix of extracellular polymeric substance which serves a protective layer by reducing antibiotic efficiency and the host immune response. Bacterial behaviour within biofilms is regulated by the phenomenon called quorum sensing (QS). QS, a population density dependent mechanism present in many bacteria, is mediated through small signal molecules called auto inducers that regulate the target gene expression which are responsible for the phenotypes essential to pathogenicity/symbiosis [2]. Among all the QS bacterial systems, Pseudomonas aeruginosa is the most intensively studied because of its fatal infections leading to the death of several thousand annually [3]. $P$. aeruginosa has two $N$-acyl-homoserine lactone (AHL) mediated (LasR/I and RhlI/R) and one alkylquinolone mediated (2-alkyl-4-(1H)-quinolone) QS systems of which AHL-mediated system was well studied $[4,5]$. In AHL mediated QS, LasI produces the N-3-oxododecanyol-Lhomoserine lactone (3-oxo-C12 HSL), activates regulator LasR and modulate the expressions of $\mathrm{Rhl}$ which encode elastase, a virulence factor. RhlI synthesize the $\mathrm{N}$ butanoyl-L-homoserine lactone (C4-HSL), which regulates rhlI and phz operon leading to the synthesis of a virulence factor, pyocyanin [6-8]. In this scenario, the QS have become the therapeutic target to attenuate bacterial virulence and thus control infection by degrading QS signals.

Syzygium aromaticum is an aromatic herb, commonly called clove, which grows in hot tropical climate [9]. Clove is used in indigestion, generalized stress, parasitic infestations, cough, toothaches, headache and blood impurities. It also acts as antiviral, antimicrobial, antiseptic and antifungal agent. According to the previous literature, $S$. aromaticum possesses inhibition of AHL short chain and virulence factors in P. aeruginosa [10-12].

In our previous study, we demonstrated the efficacy of quorum sensing inhibition (QSI), biofilm inhibition and virulence factors inhibition of S. aromatic [13]. In our continuous research, we aimed to partial purification, antiquorum and GC-MS analysis of S.aromaticum. Furthermore, in silico docking studies were performed on 
GC-MS analysed compounds in order to investigate the specific binding sites of the compounds with LasI and EsaI.

\section{MATRERILS AND METHODS}

Collection and extraction of $S$. aromaticum

S. aromaticum (buds) collected from Bengaluru, Karnataka, India, were cleaned, dried and powdered. The powdered sample was extracted with methanol at 1:10 (w/v) concentrations using Soxhlet apparatus. The extract was filtered through Whatman No.1 filter paper and the filtrate was concentrated by rotary evaporator for further use.

\section{Strains and culture condition}

Chromobacterium violaceum 12742 was procured from MTCC, Chandigarh and clinically isolated $P$. aeruginosa was collected from Bhagawan Mahaveer Jain Hospital, Bangaluru. The cultures were maintained in the Luria Bertani (LB) medium at regular intervals for further studies.

TLC and bioautographic analysis on $C$. violaceum

Methanolic extract of $S$. aromaticum was subjected to TLC. About $15 \mu$ of extract was applied to the precoated aluminum silica gel 60 F, Merck F 254. Developing solvent system used was toluene: acetone: chloroform (40:35:25). The separated spots were visualized under UV illuminator at $346 \mathrm{~nm}$ [14].
For bioautographic analysis, developed TLC plates were dried and placed on the $C$. violaceum spreader agar plate. The plates were incubated for one hour to diffuse the TLC separated fraction into the agar media. After incubation, the TLC plate was removed carefully by aseptic forceps and the agar plate was incubated at $37^{\circ} \mathrm{C}$. C. violaceum growth appeared as clear zone against purple back ground at bioactive fraction and the $\mathrm{R}_{\mathrm{f}}$ value was determined [15].

\section{Effect of bioactive fraction on biofilm formation of $P$. aeruginosa}

The effect of $S$. aromaticum bioactive fraction on the biofilm formation was determined by quantifying the biomass through microtitre plate assay [12]. The clinical pathogen was incubated in 96 well plates in the presence $(50,100,150$ and 200 $\mu \mathrm{g} / \mathrm{mL}$ ) and absence of extracts for $16 \mathrm{~h}$ without agitation. The free-floating planktonic cell were removed, washed with sterile water and stained with $0.2 \%$ crystal violet solution for $15 \mathrm{~min}$. The excess crystal violet was discarded, wells were filled with $95 \%$ ethanol and absorbance was measured at $650 \mathrm{~nm}$ using UV-visible spectrophotometer.

\section{Attenuated total reflectance infra-red (ATR-IR) analysis}


The bioactive fraction was subjected to ATR-IR (Bruker Alpha ECO-ATR spectrometer) analysis for the functional groups. $10 \mu 1$ sample was placed directly on the germanium piece of the IR spectrometer with constant pressure and wave number ranged from $4000 \mathrm{~cm}^{-1}$ to $675 \mathrm{~cm}^{-1}$. The characteristic peaks were determined according to the presence of functional groups [16].

\section{GC-MS analysis and identification of compounds}

GC-MS analysis of the bioactive fraction was performed with Thermo GC -Trace ultra Ver: 5.0, thermo MS DSQ II and equipped with column DB 5 -MS capillary standard non -polar (35 Mts x $0.25 \mathrm{~mm} \mathrm{x}$ $0.25 \mu \mathrm{m})$. The analysed patterns were interpreted by the National Institute Standard and Technology (NIST) database [17].

\section{In silicodocking studies}

In order to understand the binding sites of GC-MS analysed compounds, docking studies were performed with crystal structures of PDB ID: 1R05 (LasI) and PDB ID: 1KZF (EsaI). Chemspider was employed for drawing, displaying and characterizing the chemical as well as the 3D structures which are required for docking. All the protein molecules were pre-processed computationally by removing water molecules from the crystal structure and minimised using the MGL toolkit scripts. Theoretically published active sites for each protein molecule were identified from knowledge base. The Docking grid coordinates were plotted around $4 \mathrm{~A}^{\circ}$ radius of active pocket on protein molecule. Protein-compound docking simulation was performed using Auto Dock 4.1[18] and visualization by discovery studio. ADME molecular properties and bioactivity scores of the drug targets were calculated using Molinspiration according to Lipinski's rule for all analysed ligands [19].

\section{Statistical analysis}

All experiments were performed in triplicate. Data of each experiment showed the mean $\pm \mathrm{SE}$ and the significance of differences among was assessed using one sample t-test at $\mathrm{p} \leq 0.05$.

\section{RESULTS AND DISCUSSION}

\section{TLC Bioautography and biofilm inhibition}

Methanolic extract of S.aromaticum was separated on TLC which showed five clear bands under UV illuminator at $346 \mathrm{~nm}$. TLC bioautography of above extract exhibied the clear zone of inhibition with purple background at the bioactive fraction $\left(R_{\mathrm{f}}=0.78\right)$ (Figure 1). The earlier studies reported that crude S.aromaticum exhibited anti quorum activity against $C$. violaceum, P. aeruginosa and Aeromonas hydrophila at sub-MIC levels [12]. In the current study, 
bioactive fraction showed dose dependent biofilm inhibition on $P$. aeruginosa and maximum inhibition $(72 \% \pm 0.35)$ was found at $200 \mu \mathrm{g} / \mathrm{mL}$ (Figure 2).

\section{ATR-IR analysis}

In our previous study, ATR-IR analysis of S.aromaticum methanolic extract showed the presence of alkanes, alkynes, aldehydes, aromatics, carboxylic acids and halogen groups [13]. In the present study, ATR-IR analysis confirmed the presence of aldehydes and aromatic compounds which showed major peaks at $1709.134 \mathrm{~cm}^{-1}$ and $1260.435 \mathrm{~cm}^{-1}$ respectively (Figure $3 \mathrm{~A}$ ) indicating that the above functional groups retained in the purified bioactive fraction.

\section{GC-MS analysis}

GC-MS analysis of active fraction revealed six major compounds viz. 4-hydroxy-3methoxy-benzaldehyde, 1-phenoxy ethanol, eugenol, chavicol, p-hydroxyacetophenone and 4-azido-phenol that may account for the antibiofilm activity (Figure 3B; Table 1).

\section{In silico docking}

The molecular docking analysis was performed to understand the mechanism of compounds mediated inhibition and predicted their potential binding sites of AHL synthase. LasI is the counterpart of AHL synthase in $P$. aeruginosa which produces 3-oxo-C12-HSL, shares $31 \%$ identity $(47 \%$ homology $)$ of protein sequence with RhlI. The binding energies of compounds were calculated on LasI (Table 1). Among all, chavicol showed best pose dock with binding energy of -6.20 $\mathrm{kcal} / \mathrm{mol}$. The LasI crystal structure showed SAM binding pockets with N-terminal residue e.g. Phe 27, Arg 30 and Trp 33 and with the conserved residue of Phe 105 in acyl-chain binding site. The pose of chavicol was locked deeply into the binding site and formed three hydrogen bonds with LasI amino acids Phe 105, Thr 144 and Val 143 (Figure 4A).

In order to understand the binding properties of compounds to AHL synthase, the second docking was performed with EsaI. The binding energies also revealed that the chavicol showed best binding energy (-4.36 kcal/mol) (Table 1) and formed three hydrogen bonds with Ser 98, Arg100 and Phe101 of EsaI (Figure 4B). The amino acids, $\operatorname{Arg} 100$ and Phe101 are in the hydrophobic core of EsaI acyl-chain substrate. Docking pose revealed that chavicol bind to acyl-chain binding site in EsaI.

Earlier study reported that the binding poses of trans-cinnamaldehyde formed one hydrogen bond with Arg 30 of LasI and two hydrogen bonds of EsaI, Arg100 and Phe101 [20]. On the contrary, the present study showed that the chavicol has formed three hydrogen bonds with LasI and three 
hydrogen bonds with EsaI, indicating best interaction with targeted protein binding sites. The ADME (Molinspiration-based) of the phytochemical compounds were depicted in table 1. The compounds showed molecular hydrophobicity (miLogP) and topological polar surface areas (TPSA) which reveals the cell permeability and obeyed Lipinski's Rule of Five. For the drug lead properties or designing programs, the Lipinski's Rule has been used for the sorting of the active ligands. The compounds violating the rules (more than five H-bonds donors, H-bond acceptors, a molecular weight (MW) greater than 500, and $\log \mathrm{P}$ value greater than five) may show poor absorption or permeation [21 and the compounds in the present study are in the accordance with Lipinski’s Rule.

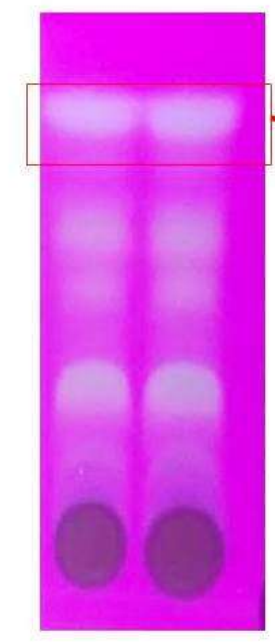

A

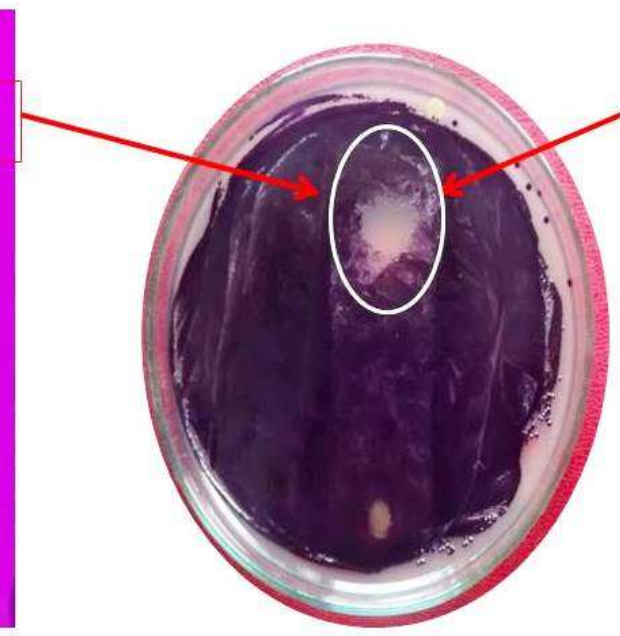

B

Figure 1: TLC separation and bioautography of S.aromaticum extract.

A) TLC separation of S.aromaticum extract; B) TLC bioautography of S.aromaticum extract on C. violaceum

100

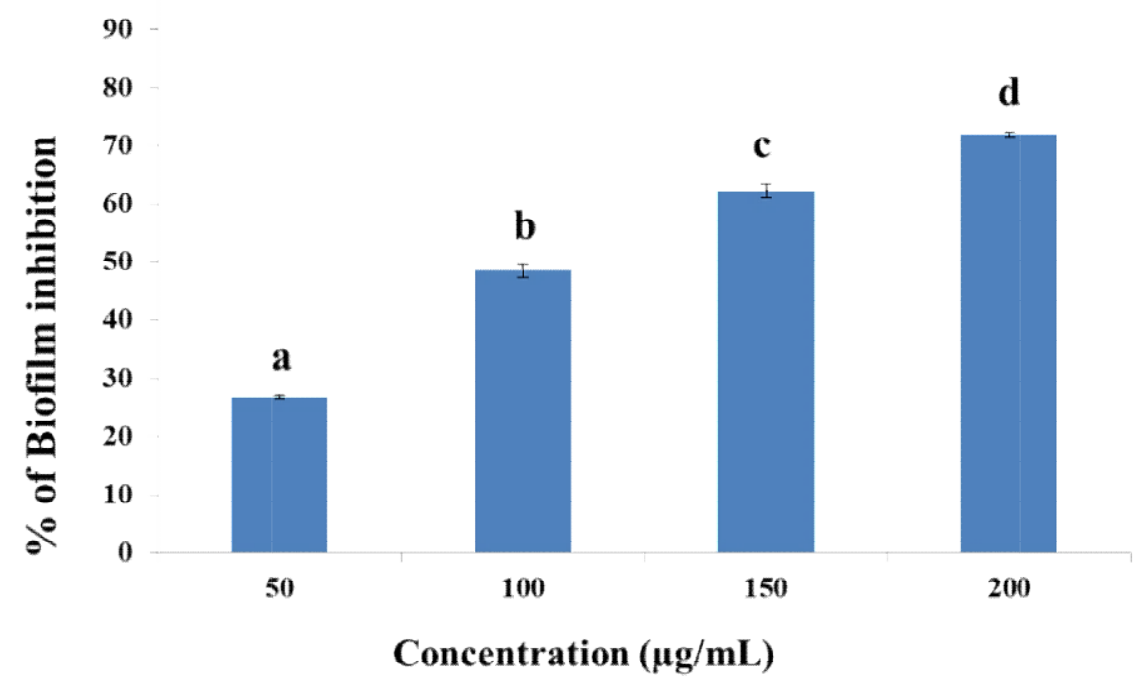

Figure 2: Biofilm inhibition of bioactive fraction of S.aromaticum 
(A)

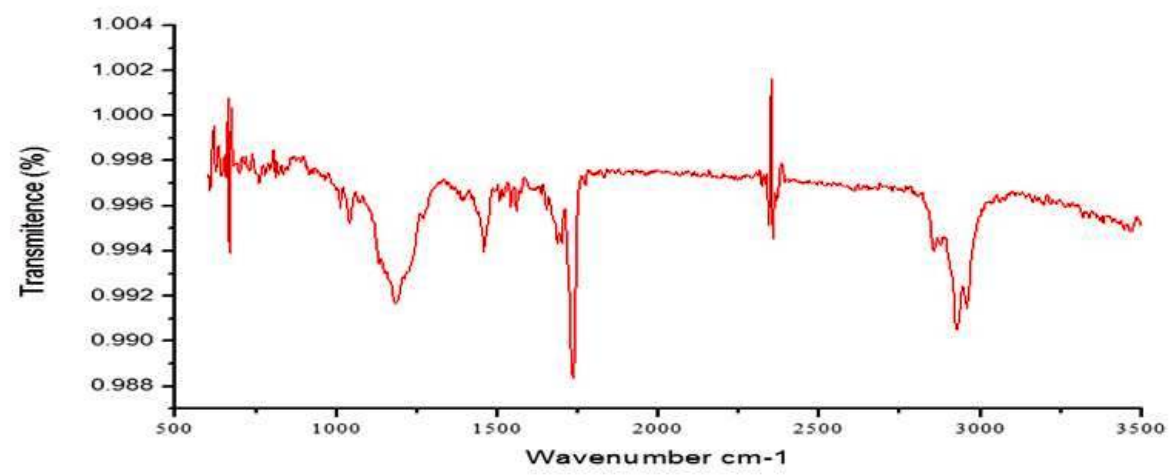

(B)

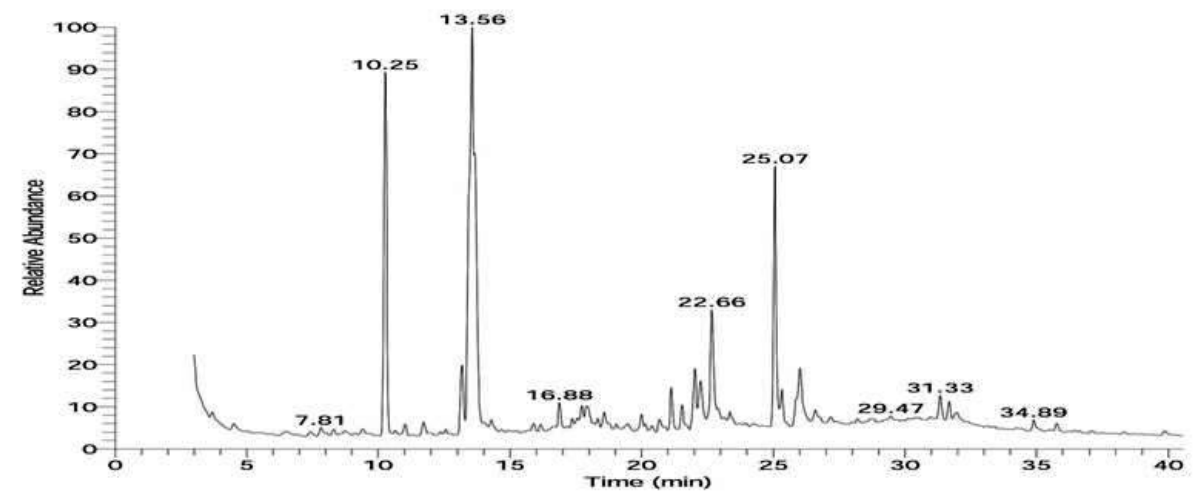

Figure 3: (A) ATR-IR and (B) GC-MS analysis of bioactive fraction.

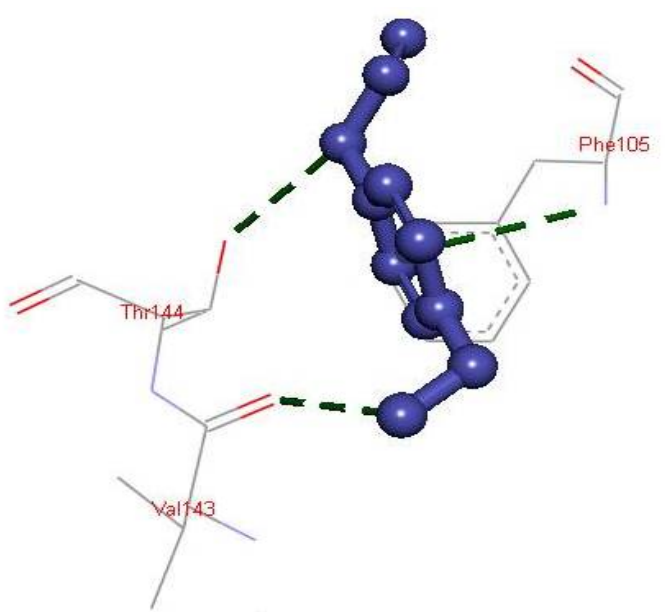

A

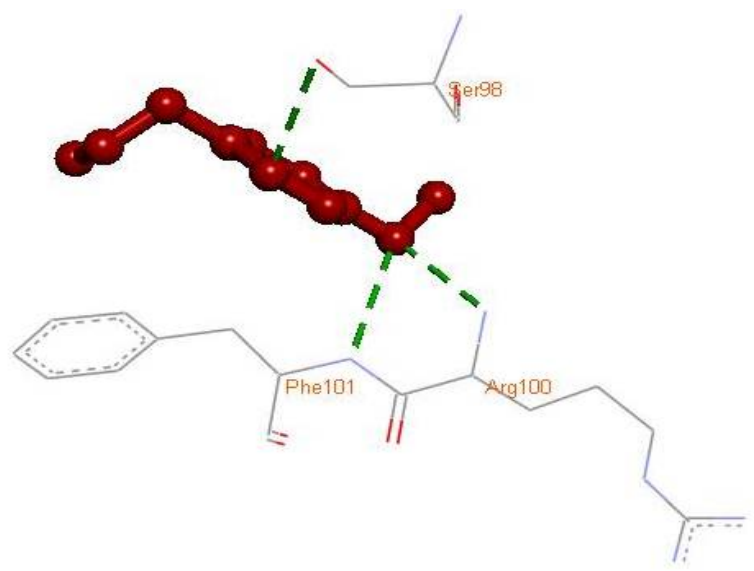

B

Figure 4: Binding and hydrogen bonds formation of 5-phenyldecane with (A) LasI and (B) EsaI 
Table 1: Docking scores of GC-MS compounds with active site of $P$. aeruginosa quorum sensing regulators and their ADME analysis

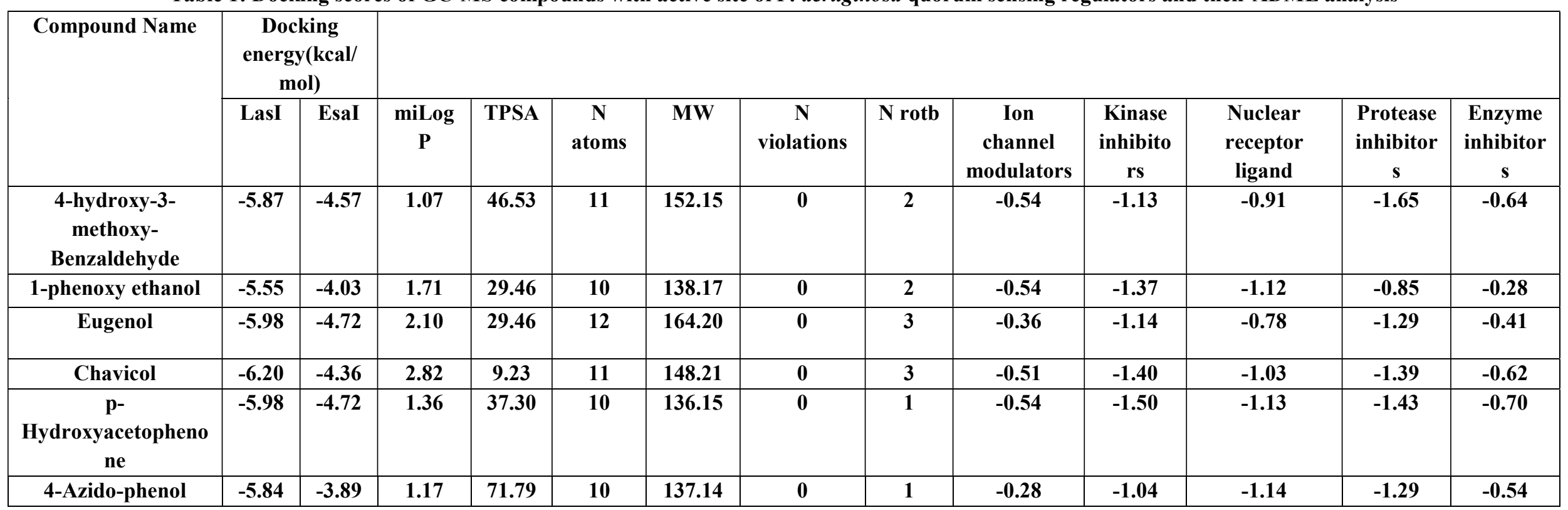




\section{CONCLUSION}

In conclusion, our results demonstrated that the S.aromaticum bioactive fraction has quorum quenching ability. The in silico analysis supported the in vitro study which might lead to in vivo establishment in future.

\section{ACKNOWLEDGEMENT}

The authors are grateful to the Jain University for providing infrastructure for carrying out the research work. We thank Dr. Geetha V Bhat, Microbiologist and Infection control officer, Bhagwan Mahaveer Jain Hospital, Bengaluru for providing $P$. aeruginosa strain. We also thank Dr. Geetha Balakrishna, Director, CNMS, Jain University, for ATR-IR spectra analysis.

\section{REFERENCES}

[1] Lewis K, Persister cells, dormancy and infectious diseases, Nature Reviews Microbiology, 5, 2007, 48-56.

[2] Nusbaum AG, Kirsner SR, Charles AC, Biofilm in dermatology, Skin Therapy Letter,17, 2012, 7-12.

[3] Sudha C, Everett PG, An evolving perspective on the Pseudomonas aeruginosa orphan quorum sensing regulator QscR, Frontiers in
Cellular and Infection

Microbiology, 4, 2014, 1-7.

[4] Williams P, Camara M, Quorum sensing and environmental adaptation in Pseudomonas aeruginosa: A tale of regulatory networks and multifunctional signal molecules, Current Opinion in Microbiology, 12 , 2009, 182-191.

[5] Antunes LCM, Ferreira RBR, Buckner MMC, Finlay BB, Quorum sensing in bacterial virulence, Microbiology, 156, 2010, 2271-2282.

[6] Shirtliff ME, Mader JT, Camper AK, Molecular interactions in biofilms, Chemistry \& Biology, 9, 2002, 859-871

[7] Wagner VE, Bushnell D, Passador L, Brooks AI, Iglewski $\mathrm{BH}$, Microarray analysis of Pseudomonas aeruginosa quorum-sensing regulons: effects of growth phase and environment, Journal of Bacteriology, 185, 2003, 20802095.

[8] Gonza'lez JE, Keshavan ND, Messing with bacterial quorum sensing, Microbiology and Molecular Biology Reviews, 70, 2006, 859-875. 
[9] Debjit B, Kumar KPS, Yadav A, Srivastava SS, Paswan S, Dutta AS, Recent trends in Indian traditional herbs Syzygium aromaticum and its health benefits, Jourmal of Pharmacognosy and Phytochemistry, 1, 2012, 6-17.

[10] Khan MSA, Zahin M, Hasan S, Husain FM, Ahmad I, Inhibition of quorum sensing regulated bacterial functions by plant essential oils with special reference to clove oil, Letters in Applied Microbiology 49, 2009, 354-360.

[11] Krishnan T, Wai FY, Kok GC, Inhibition of quorum sensingcontrolled virulence factor production in Pseudomonas aeruginosa PAO1 by Ayurveda spice Clove (Syzygium aromaticum) bud extract, Sensors, 12, 2012, 4016-4030.

[12] Husain FM, Iqbal A, Mohammad A, Qudsia T, Influence of clove oil on certain quorum- sensing- regulated functions and biofilm of Pseudomonas aeruginosa and Aeromonas hydrophila, Journal of Biosciences, 38, 2013, 835844.
[13] Mutungwa W, Alluri N, Majumdar M, Anti-quorum sensing activity of some commonly used traditional Indian spices, International Journal of Pharmacy and Pharmaceutical Sciences, 7, 2015, 80-83.

[14] Alluri N, Majumdar M. Phytochemical analysis and in vitro antimicrobial activity of Calotropis gigantea, Lawsonia inermis and Trigonella Foecumgraecum, International Journal of Pharmacy and Pharmaceutical Sciences, 6 (4), 2014, 524-527.

[15] Irena MC, Wioleta J, TLCDirect bioautography as a high throughput method for detection of antimicrobials in plants, Journal of Chromatography, 2, 2015, 225-238.

[16] Beatriz RL, Attenuated total reflectance spectroscopy of plant leaves: a tool for ecological and botanical studies, New Phytologist, 172, 2006, 305-318.

[17] Alluri N, Majumdar M, In vitro anticancer potential and GC-MS analysis of Drima nagarjunae, an endangered medicinal plant, Bangladesh journal of 
Pharmacology, 10, 2015, 303307.

[18] Sohaibani SA, Murugan K, Anti-biofilm activity of Salvodora persica on cariogenic isolates of Streptococcus mutans: in-vitro and molecular docking studies, Biofouling, 28, 2012, 29-38.

[19] Ertl P, Database of bioactive ring systems with calculated properties and its use in bioisosteric design and scaffold hopping, Bioorganic \& Medicinal Chemistry, 20, 2012, $5436-5442$.

[20] Chien YC, Thiba K, Hao W, Chen Y, Wai-Fong Y, Yee-
Meng $\mathrm{C}$, Li Ying T, Teik, MC, Kok-Gan C, Non-antibiotic quorum sensing inhibitors acting against $\mathrm{N}$-acyl homoserine lactone synthase as druggable target, Scientific Reports, 4, 2014, 1-8.

[21] Gonçalves CJ, Lenoir AS, Padaratz P, Corrêa R, Niero R, Cechinel-Filho V, Campos BFD, Benzofuranones as potential antinociceptive agents: Structure activity relationships, European Journal of Medicinal Chemistry, $56,2012,120-126$. 